\title{
A new method to evaluate order and accuracy of inaccurately and incompletely reproduced movement sequences
}

\author{
Johannes Drever • Andreas Straube • Thomas Eggert
}

Published online: 12 November 2010

(C) Psychonomic Society, Inc. 2010

\begin{abstract}
Studying imitation learning of long sequences requires the evaluation of inaccurately and incompletely reproduced movement sequences. In order to evaluate the movement reproduction, it has to be assigned to the original stimulus. We developed an assignment algorithm that considers the Spatial Neighborhood and Order of reproduction (SNOA). To evaluate the features of this analysis it was applied to human performance during learning of long pointing sequences under two conditions: stimulus-guided reproduction with high spatial accuracy and imitation learning with low spatial accuracy. The results were compared with a simple assignment considering Spatial Neighborhood only (SNA) and with a Manual Assignment (MA). In the stimulus-guided reproduction the error measures did not differ between the algorithms. In contrast, with imitation learning, SNOA and MA generated higher estimates of order and omission errors than SNA. The results show that SNOA can be used to automatically quantify the similarity of both movement structure and metric information between long target sequences and inaccurate and incomplete movement reproductions.
\end{abstract}

Keywords Sequence learning · Imitation learning · Contour matching

J. Drever $\cdot$ A. Straube $\cdot$ T. Eggert

Department of Neurology, Ludwig Maximilian University,

Munich, Germany

A. Straube

e-mail: Andreas.Straube@med.uni-muenchen.de

T. Eggert

e-mail: eggert@1rz.uni-muenchen.de

J. Drever $(\bowtie)$

Department of Neurology, Klinikum Grosshadern,

Marchioninistr. 23,

81377, Munich, Germany

e-mail: drever@1rz.uni-muenchen.de

\section{Introduction}

Human behavior comprises many skills, such as writing, playing the piano or dancing a waltz. All these skills are composed of sequential actions. It is very likely that these complex movement sequences are not learned at the very first attempt, but that a movement plan gradually builds up. Since Lashley's classic work (1951) there has been agreement that the order of movement elements may be independent of the nature of the movement actions. Many paradigms to investigate movement sequences have emerged in the past decades. In these paradigms, subjects are either challenged to reproduce short sequences by saccadic eye movements or button presses (Ditterich, Eggert, \& Straube, 1998; Sternberg, Monsell, Knoll, \& Wright, 1978), or long sequences that are trained as reactive movements to visible stimuli and become internally generated after learning (Ghilardi, Moisello, Silvestri, Ghez, \& Krakauer, 2009; Hikosaka, Rand, Miyachi, \& Miyashita, 1995; Wilde \& Shea, 2006). These paradigms can be divided into imitation learning, where a stimulus is presented and imitated after presentation, and stimulus-guided learning, where the reproduction occurs concurrent with the presentation. Evaluating the reproduction performance in these paradigms requires the assignment of reproduced positions to target positions; a task that differs widely in complexity.

When the stimulus is reproduced by discrete button presses, the assignment of reproductions to targets is trivial (e.g., Hikosaka et al., 1995; Sternberg et al., 1978). Assigning continuous sequence reproduction is more problematic, especially in the presence of order errors and omissions. Consider the task illustrated in Fig. 1. In this task a long sequence of stimuli is presented and has to be reproduced in the absence of any stimuli after presentation. In this task the pointing movements have to be assigned to 
A

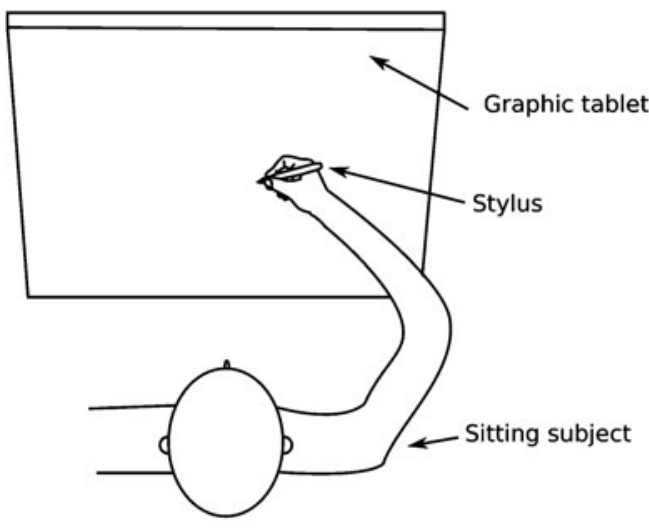

Fig. 1 Illustration of the learning task. A) The subjects sat in front of a graphic tablet that featured a display for stimulus presentation. The presented target locations were reproduced by pointing with a stylus. B)

the original locations of the stimulus. For short sequences, this problem can be solved adequately by a simple nearest neighbor assignment (Ditterich et al., 1998). Ambiguous trials that contain order errors or omissions are simply omitted for further analysis.

Most learning paradigms for long sequences are stimulus-guided, thereby allowing an accurate reproduction. With such an accurate reproduction the assignment can be implemented by a nearest neighbor search.

In contrast, if the number of reproduced positions is much smaller than the number of target positions, or if the reproduction accuracy is low with respect to the target distances, these methods are not suitable, e.g., during the early phase of imitation learning of long pointing sequences. This is probably one reason why sequence reproduction under these conditions is rarely studied. Inaccurate pointing reproductions are also expected when imitation learning of sequential movements is examined in patients with movement disorders (cerebellar lesions) or memory impairments (hippocampal lesions). Studies focusing on the theoretical issue of how metrical and order information is acquired have focused on stimulus-guided learning (Ghilardi et al., 2009; Wilde \& Shea, 2006). Investigating the frequency of order errors during the early phase of imitation learning may give further insight into these theoretical issues.

We investigated imitation sequence learning in a paradigm in which the sequence was internally generated without guiding stimuli. To solve the assignment problem we developed a new algorithm that takes the order of reproduction into account. This Spatial Neighborhood and Order considering Assignment (SNOA) is composed of the longest continuous chunks that minimize the mean
The training phase of a trial. Twenty target positions were presented one after another with an inter-stimulus interval of $1 \mathrm{~s}$. The disappearance of the last cross was the go-signal for the reproduction

distance between targets and assigned reproductions. ${ }^{1}$ To illustrate the features of the assignment it was contrasted with the nearest neighborhood assignment, which does not consider the order of reproduction (Spatial Neighborhood considering Assignment, SNA). These two assignments were first compared under a condition in which targets were visible during reproduction and pointing accuracy was high. In this case SNA should achieve a good assignment and SNOA should not deviate from this assignment. In the second condition the targets were reproduced in the absence of any stimuli. In this case inaccurate and incomplete reproductions have to be assigned to the target sequence during learning. We investigated whether the SNOA and SNA assignments deviated under these circumstances and evaluated the plausibility of the assignments by comparison with manual assignments.

\section{Material and methods}

\section{Procedure}

Ten healthy subjects participated in the experiments (mean age: 33, range: $28-51$ years). They gave informed consent before participating in the study. The study was performed in accordance with the Declaration of Helsinki and was approved by the local ethics committee. The subjects sat in a dark room in front of a graphic tablet that featured an integrated computer display (Fig. 1a, WACOM Cintiq

\footnotetext{
${ }^{1}$ The MATLAB implementation of SNOA can be downloaded at http://www.nefo.med.uni-muenchen.de/ teggert/SNOA/JBRM.tar.gz.
} 
$21 \mathrm{UX}, 43.2 \times 32.4 \mathrm{~cm})$. The viewing distance between subject and display was about $30 \mathrm{~cm}$. A single experimental trial consisted of a presentation phase and a reproduction phase. During the presentation phase, a long pseudorandom sequence of 20 positions was presented with a white cross (size: $1 \times 1 \mathrm{~cm}$ ) that stepped through the sequence with a fixed inter-target interval of $1 \mathrm{~s}$ (Fig. 1b). Subjects were instructed to track the target with eye movements, but they were not allowed to perform manual pointing during this presentation phase. After all 20 targets had been presented, the initial position was shown for $1 \mathrm{~s}$, and the cross disappeared. The disappearance of the cross was the go-signal to start the reproduction. Subjects were instructed to reproduce as many target positions as accurately as possible by manual pointing with a stylus on the graphic tablet. They were asked to lift the pen between successive pointing movements. They were instructed to produce the sequence in the correct order, but were allowed to continue production after omissions or order errors occurred. The subjects indicated the end of the reproduction by pressing a button when they could not recall any further target positions. The reproduction was performed under two different conditions. In the first condition all targets were simultaneously visible ("visually guided'; V). As each target was reproduced, its shading changed in order to provide the subject with information about which targets had been already reproduced. In the second condition, the sequence had to be reproduced on a blank screen ('imitation'; I). All events, target positions, pointing positions (accuracy $<1 \mathrm{~mm}$ ), and the button presses were recorded on a central recording system REX (Hays, Richmond, \& Optican, 1982) for later analysis.

The sequence length was chosen in such a way that a complete reproduction on the first attempt was impossible, since the focus of the study was to investigate inaccurate and incomplete sequence reproduction. In one experimental session, 25 trials (alternating presentation and reproduction) were repeated with the same sequence in order to allow subjects to learn the whole sequence.

The target sequences contained only a minimal amount of geometrical regularity since the target positions were chosen randomly from a quadratic area $(28 \times$ $28 \mathrm{~cm})$. Some constraints were introduced in order to avoid spatial clustering of target positions: The minimal distance between targets was $4 \mathrm{~cm}$. Only one additional position was allowed within a radius of $6 \mathrm{~cm}$ around each target. Further interactive inspection assured that target sequences used for training did not contain apparent geometrical features such as three points on a line or four points forming a rectangle. In both conditions subjects learned a new sequence that they had not seen before.
Analysis

The first step of error analysis was the assignment of reproductions to the original target locations for each trial. SNA was performed as follows: for each target the nearest reproduction was found and assigned to the corresponding target. Reproductions that were further away from any target than the minimal distance between targets were not assigned.

SNOA was performed by an algorithm in several steps. The assignment was composed of continuous subsequences of target positions to which reproductions were assigned. These subsequences are called 'chunks.' In summary, the algorithm first searched for the longest chunk. This chunk had to minimize assignment costs, as defined later. When this chunk was found, the reproductions and targets belonging to this chunk were removed and the same procedure was applied to the remaining target and reproduction locations until no further assignments were found.

Chunks were found with a linear-order-preserving assignment algorithm (Scott \& Nowak, 2006). The algorithm performed an order-preserving assignment of reproductions to target positions by minimizing assignment costs. These costs were defined as the sum of the distances between assigned reproductions and targets and the number of nonassigned targets weighted by a factor $\varepsilon .^{2}$ Thus, in the linearorder-preserving assignment algorithm, a reproduction with a distance larger than $\varepsilon$ from the nearest target was never assigned.

To find the longest chunk the linear-order-preserving assignment was applied to all continuous subsequences of targets with a given length. The algorithm started with the full target sequence and proceeded systematically to all shorter subsequences until at least one of these continuous target chunks was completely assigned by the Scott-Nowak algorithm. This assignment defined the longest chunk. If several chunks of the same length were completely assigned, the longest chunk was defined by the matching with the minimal costs.

After the longest chunk was found, the assigned targets and reproductions were removed from the original target sequence and reproduction sequence respectively. Then the algorithm recursively started to search for the next longest chunk. This procedure was repeated until no further assignments were found.

Note that even though this algorithm preserves the order within each of the chunks, it is able to detect any order errors between chunks. This strategy is computationally

\footnotetext{
${ }^{2} \varepsilon$ was chosen to be the minimum distance between targets $(5.5 \mathrm{~cm})$. Note that $\varepsilon$ could also be chosen adaptively, e.g. by the asymmetric Hausdorff distance defined by the maximum of minimal targetreproduction distances.
} 
much more efficient than any combinatorial optimization procedure because of the large number of possibilities to assign reproductions to targets. The linear-orderedassignment algorithm adopted from Scott \& Nowak (2006) is a generalization of the efficient dynamic programming algorithm proposed by Levenshtein (1966). Thus, the assignment can be solved with little expenditure of time on a modern computer.

The SNOA assignment was developed to mimic the tendency of human observers to incorporate the order of recall when performing a subjective assignment. To confirm this, the 25th reproduction of the imitation condition (I) of eight subjects was manually assigned by four human evaluators (Manual Assignment: MA). The evaluators were familiar with the experiment but had no extensive training on assignment. All evaluators were told that the subjects had three different error possibilities: number of omissions, accuracy error and order errors. The target sequence and reproduction sequence were illustrated by static line drawings with numbered vertices, superimposed on a computer monitor. Each reproduction vertex either had to be classified as an outlier or had to be assigned to a target vertex in a bijective manner.

Based on SNA, SNOA or MA, three different error measures were defined. The omission error was defined by the number of non-assigned targets. The accuracy error was defined by the mean distance between assigned targets and reproductions. The order error was defined by the number of transpositions that was needed to sort the reproductions into a linear ascending list with a shell sort algorithm. The shell sort algorithm achieves sorting with a very small number of pairwise exchanges (Pratt, 1972). To simplify references to an error based on a specific assignment method, its name will hereafter be attached as a prefix to the error name, e.g., SNA accuracy error is the accuracy error evaluated based on SNA.

\section{Results}

General observations

Generally, no subject was able to reproduce the sequence after the first trial, either in the visually guided or in the imitation condition. Most subjects started with the reproduction of only few targets, which in general were at the beginning of the sequence and increased the number of reproductions after each learning trial. In this way the sequence was learned from the beginning to the end. The time until subjects were able to reproduce the whole sequence varied from subject to subject and was much shorter in the visually guided condition. Note that the accuracy errors and order errors at the beginning of learning would have been much higher if subjects had been forced to reproduce the whole sequence.

Subject performance during visually guided reproduction

The resulting number of omissions, the accuracy error and the number of order errors under visually guided reproduction conditions (V) are shown in Fig. 2. Under this condition the error evaluation based on SNA and on SNOA did not differ substantially. In this paragraph, the reported subject performance is based on SNOA, and differences between SNA and SNOA are reported in the next paragraph. The main learning progress was reflected in the number of omissions. Subjects started with 16 omissions on average, and the performance saturated at a few omissions after 11 trials $(0.2 \pm 0.4$ omissions $)$. Thus, subjects were able to reproduce the whole sequence after the 11th trial. Pointing to the visible target locations was very accurate throughout the whole experiment $(0.3 \pm$ $0.1 \mathrm{~cm})$. Before the tenth trial about one order error occurred on average. After the tenth trial, order errors occurred very rarely $(0.5 \pm 0.5$ omissions).

\section{Differences between SNA and SNOA}

Before that tenth trial, the number of omissions did not differ between the measures. After the tenth trial, SNOA indicated slightly fewer omissions than SNA (Fig. 2a). The average number of omissions after the tenth trial was $1.2 \pm$ 0.4 (SNA) and $0.5 \pm 0.4$ (SNOA). The accuracy of pointing did not change over time when evaluated with the SNA algorithm (Fig. 2b). The SNOA algorithm indicated a small temporary decrease of the accuracy during trials 5-6. The number of order errors was low throughout the whole experiment and was only slightly lower for SNOA in trials 5-6. Otherwise, the number of order errors did not differ between SNA and SNOA (Fig. 2c), which was expected for the visually guided condition.

An illustrative example of the differences between SNA and SNOA

The differences between SNA and SNOA are illustrated with an example in Fig. 3. It shows a detail of the reproduction and assignment of the 6th trial of one subject. In this sequence, target 14 is close to target 5 . The subject reproduced the sequence 4-5-6 correctly, but accidentally the reproduction of target 5 is spatially closer to target 14 than to target 5. SNA assigns reproduction 5 to the spatially closer target 14, whereas SNOA correctly assigns reproduction 5 to target 5 . Thus, SNOA judges reproduction 5 as an inaccurate reproduction of target 5 rather than an accurate reproduction of target 14 . In contrast, SNA judges 


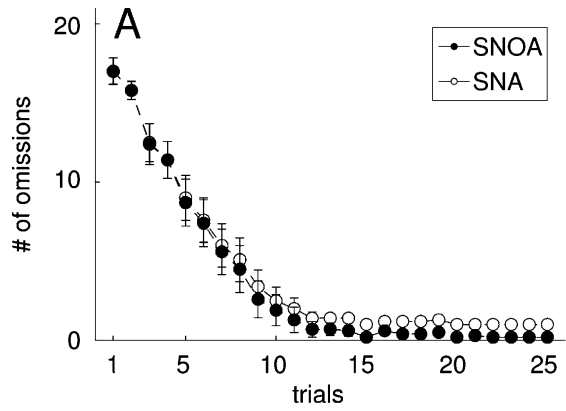

Fig. 2 The error measures based on SNA and SNOA do not differ in the visually guided condition $(V)$. The mean of the error measure $( \pm$ SE) A) number of omissions, B) accuracy error and C) number of

the reproduction as a confusion of target 14 with target 5 . The frequency with which SNOA judged three consecutive reproductions as a continuous sequence and SNA did not was 2.09 times per trial on average.

Subject performance during imitation

The number of omissions, the accuracy error and the number of order errors when subjects reproduced the sequence on the blank screen (condition I) are shown in Fig. 4. In this paragraph, the reported subject performance is based on SNOA. The differences between SNA and SNOA are reported in the next paragraph. The main learning progress was reflected in the number of omissions. Subjects started with 16 omissions on average, and the performance saturated at about 2 omissions after 15 trials. In condition I the number of trials needed to reproduce the whole sequence was larger and when learning saturated the number of omissions was higher than in condition $\mathrm{V}$ [number of omissions in the last trial: $1.5 \pm 1.6$ (I), $0.2 \pm$ 0.4 (V), Fig. 4a]. The accuracy error was constant at about

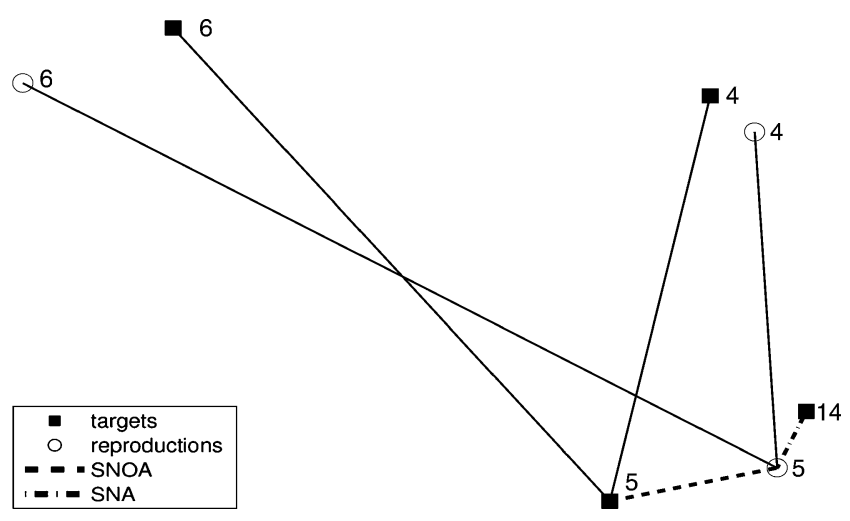

Fig. 3 Illustration of the assignment problem with inaccurate and incomplete reproductions. Targets 5 and 14 are spatially close. The reproduction of target 5 is closer to target 14 than to target 5 . In this case SNA produces a false order error, whereas SNOA favors correct order at the expense of the accuracy error that becomes only slightly larger

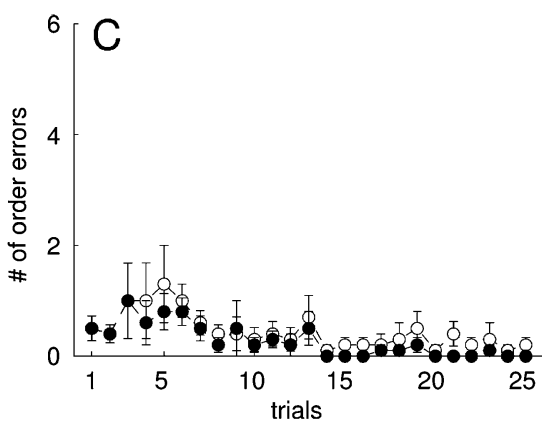

order errors. The analysis is either based on SNOA (filled circles) or SNA (circles). The error measures are pooled across ten subjects

$2 \mathrm{~cm}$ throughout the whole experiment. Pointing was much more inaccurate than in condition V (Fig. 4b). On average, the number of order errors started below one, increased to two and finally decreased again to below one order error (Fig. 4c).

\section{Differences between SNA and SNOA}

The number of omissions was higher when the analysis was based on SNA. The final performance saturated at five omissions as opposed to the two omissions indicated by the SNOA-based analysis. The accuracy error was almost constant with both assignments, but was lower for the SNA-based analysis. The number of order errors started similarly to the SNOA-based order errors, but instead of a final decrease, the SNA assignment indicated that the number of order errors increased and reached a final value of more than two.

\section{Comparison of manual assignment, SNA and SNOA}

The comparison between the manual assignment SNA and SNOA is presented in Fig. 5 for eight individual subjects. Compared to MA and SNOA, SNA produces more order and omission errors in each of the eight subjects. For all subjects the SNA number of omissions was higher than MA and SNOA omissions. In four subjects the number of omissions did not differ between SNOA and MA, and in four subjects it was higher in SNOA than in MA. The mean number of omissions across the eight subjects was $0.7 \pm 0.3$ (MA), $4.5 \pm 1.8(\mathrm{SNA})$ and $1.5 \pm 1.6$ (SNOA).

The SNA number of order errors was much higher than MA for six subjects and did not differ for the other two subjects. The SNOA number of order errors differed from MA omissions in only two subjects. The mean number of order errors across the eight subjects was $0.3 \pm 0.3$ (MA), $3.1 \pm 2.5$ (SNA) and $0.7 \pm 0.1$ (SNOA).

The accuracy error was nearly identical for all assignments [mean accuracy error across subjects and evaluators: 

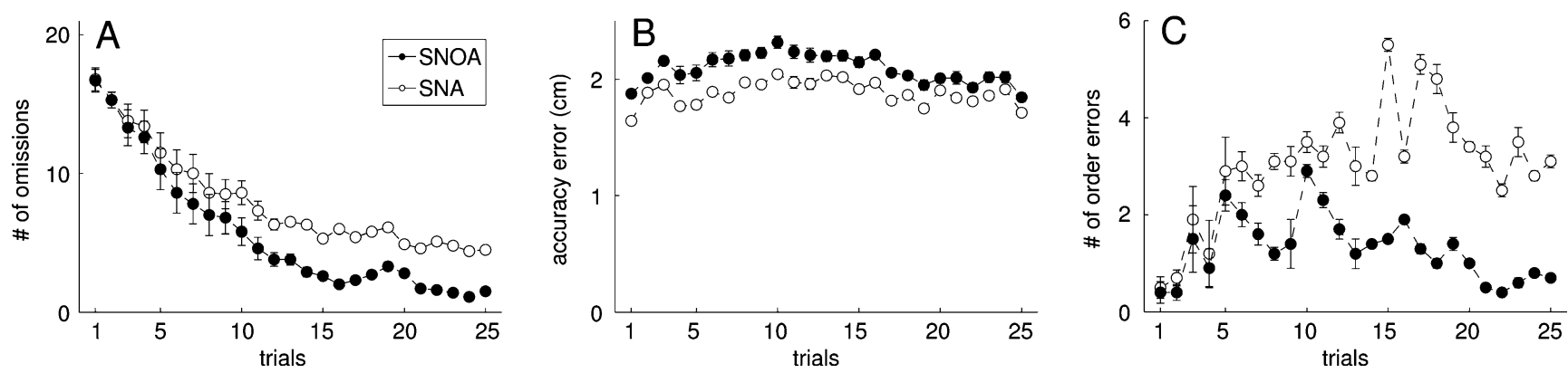

Fig. 4 SNA produced higher estimates of omissions and order errors than SNOA. Time courses of the mean error measures ( \pm SE) in the imitation learning condition (condition I), pooled across ten subjects. The analysis is either based on SNOA (filled circles) or SNA (circles)

$2.0 \pm 0.1(\mathrm{MA}), 1.7 \pm 0.2(\mathrm{SNA}), 1.8 \pm 0.3(\mathrm{SNOA})] . \mathrm{In}$ summary, SNOA mimicked MA more closely than SNA. The strongest differences between SNA and MA emerged in the overestimate of the number of order and omission errors when the evaluation was based on SNA (black and gray bars in Fig. 5, MA-SNA).

\section{Discussion}

The new order considering assignment algorithm (SNOA) was successfully applied to a learning paradigm that required subjects to learn and produce a long sequence of continuous target positions. The algorithm was compared under two conditions to the nearest neighbor assignment (SNA) that does not take reproduction order into account. In the condition with visually guided reproduction (condition V), pointing accuracy was high. The error measures obtained from SNOA and SNA showed no qualitative difference. In contrast, during imitation learning (condition I) with low spatial accuracy, SNA

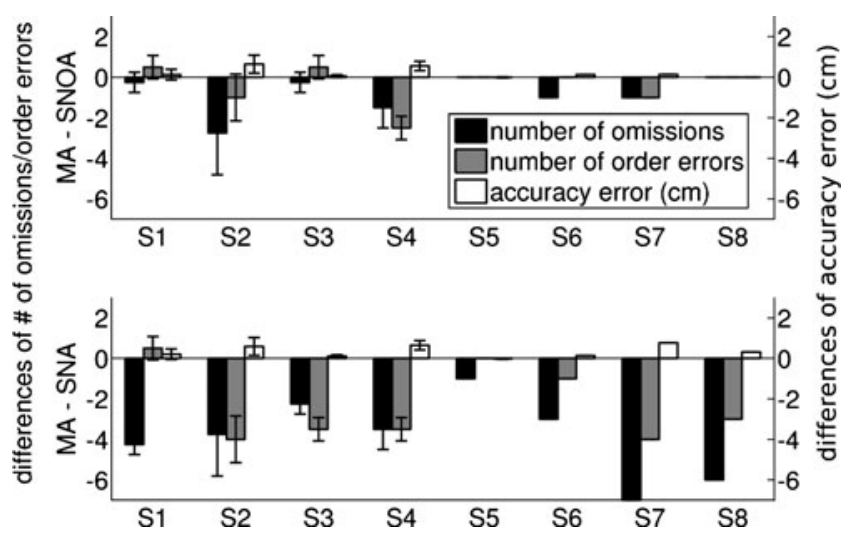

Fig. 5 Differences between manual assignment $(M A)$ and assignment considering spatial neighborhood and order (SNOA) and assignment considering spatial neighborhood only $(S N A)$. The differences in number of omissions, number of order errors and accuracy error are shown for eight individual subjects. Whiskers indicate the standard deviation across the four manual assignments. For the last four subjects all evaluators agreed in their MA. The number of order and omission errors were clearly overestimated by SNA compared to MA generated a higher number of omissions and order errors than the manual assignment MA and SNOA.

Features of the assignment algorithm

The assignment of reproductions to target positions is the crucial step in the analysis of the reproduction performance. The difficulty in this task is to differentiate among inaccurate reproductions, order errors and pointing movements that cannot be assigned to any target. In general, the decision whether an incorrect reproduction is classified as an accuracy error with correct order or as an order error with high spatial accuracy can only be solved by a heuristic assignment strategy. The simplest solution to this assignment problem is the nearest neighbor assignment (SNA). This assignment induces a high number of order errors that increase over trials without guiding stimuli (Fig. 4c). Studies in repeated serial recall of word lists showed that order errors tend to decrease during learning (Klein, Addis, $\&$ Kahana, 2005). This suggests that the increasing number of order errors quantified by SNA is rather an artifact of the assignment than a feature of the learning process.

Another commonly used alternative to evaluate reproduced movements with respect to the original is the socalled "average path error," which is defined by the mean accuracy error resulting from purely order-based assignment (e.g., Basdogan, Kiraz, Bukusoglu, Varol, \& Doğanay, 2007; Schwager, Anderson, Butler, \& Rus, 2007). With this method, a reproduction skipping one or several targets and all subsequent reproductions will be inappropriately assigned and will lead to an inappropriate increase of accuracy error. Thus, this method is, in contrast to SNA, not suitable to evaluate discontinuous reproductions that are subject to omission errors.

SNOA can be considered as a compromise between SNA and purely order-based assignment since SNOA takes the order of reproduction into account by favoring longer continuous chunks reproduced with slightly lower accuracy over isolated assignments with high accuracy. This preference can easily be strengthened by increasing the parameter $\varepsilon$ (see 
Methods). This strategy is reasonable under the assumption that spatial information is stored as order preserving chunks rather than as isolated positions. It is important to note that this assignment is able to detect any sort of order error, since the linear ordered assignment could detect the reproduction of a target chunk in any location within the reproduction sequence. Moreover, for the same reason, it also works well independently of whether omissions occur at the beginning, the end or anywhere in the target sequence. It can also handle "explorative" reproductions that are not related to any memorized target location. The algorithm does not assign reproductions that are too far away from the nearest target (threshold $\varepsilon$ ) or reproductions for which all targets within this $\varepsilon$-neighborhood are parts of longer chunks. These non-assigned reproductions were classified as explorative reproductions.

It is important to note that the SNOA cannot be considered as an objective standard for evaluating any type of sequential movement reproduction because there is no direct knowledge of the intended movements. However, the SNOA allows inaccurate and ordered reproduction to be favored over accurate and incorrectly ordered reproduction in a well-defined way (by modifying the parameter $\varepsilon$ ). This feature is essential for evaluating inaccurate and incomplete reproductions, since lack of this feature under such circumstances must lead to systematic overestimation of pointing accuracy simultaneously with an inadequately increase of apparent "order errors" (see Fig. 3).

The comparison to the manual assignment showed that SNOA performs a more reasonable assignment than SNA when pointing is inaccurate. When targets are visible and SNA is very likely to assign the correct target, the SNOA assignment is nearly identical to SNA (Fig. 2). Small differences can be found in early trials in the accuracy error. These differences are related to inaccurate reproductions that are accidentally close to targets with large order distances. These targets are assigned by SNA, ignoring the large order distance. SNOA detects the structural similarity between the target and reproduction subsequences and leads to a more plausible assignment.

\section{Features of the error measures}

Many theories in motor learning suggest that the structure of a movement sequence is determined independently of the metrical features of the movement (see Shea \& Wulf, 2005 for a review). The acquisition of movement structure is quantified with a categorical measure, and the metrical features are quantified by a continuous measure. With our assignment algorithm the number of omissions can be used to characterize structural similarity between target and reproduction, and the accuracy error can be used to characterize the metrics of the movements. This interpretation is justified under the condition that ordered reproductions of movements are more likely to occur than nonordered reproduction. In this case, and if the assignment is problematic, an assignment that considers the order, such as SNOA, will always give better results than order-ignoring assignments like SNA. Consequently, the time course of accuracy error and the number of omissions can also be used to quantify the learning progress of metrical features and movement structure, respectively. Additionally, it is possible to quantify whether errors occur in the acquired movement structure by the number of order errors.

The error measures are not fully determined by the assignment. The number of omissions can be defined as a strictly positional, relative order or lenient scoring (Addis \& Kahana, 2004). The strictly positional scoring only counts target positions that are recalled in the correct order. The relative order scoring also includes target positions that have the correct predecessor. The lenient scoring counts all target positions that were assigned. In the approach tested here the number of omissions was chosen to be a lenient scoring, because SNOA allowed simultaneous quantification of order errors. The same alternatives (strictly positional, relative or lenient) also exist for the selection of the targets contributing to accuracy error. The problem with the lenient selection applied here is that accuracy measures evaluated on erroneously assigned targetreproduction pairs lead to a systematic overestimate of the accuracy. In the SNOA assignment it is less likely that a reproduction is assigned erroneously to nearby targets than in SNA. Therefore, the lenient selection including the nonordered assignments in the accuracy measure is less errorprone than with SNA.

Specificity of the algorithm to imitation learning of long position sequences

Learning and memory of movement sequences have been subject to investigation in several studies. The task presented in this article has the unique feature that a long sequence of unconstrained pointing movements is learned with a delayed reproduction. Generally the application of SNOA is only favorable in tasks that require the assignment of inaccurate and incomplete pointing sequences. This section discusses the application of SNOA to other tasks.

The buildup of the structure and metric information of movement sequences has been quantified simultaneously by Ghilardi et al. (2009) in a stimulus-guided learning paradigm. In stimulus-guided paradigms learning is observed as a shift from stimulus-driven to internally-driven responses. Under these conditions the movements are always directed to the correct target, and pointing accuracy is very high. Thus, the assignment problem does not occur, and a nearest neighbor 
assignment is sufficient. It has been shown that delayed reproduction resulted in superior acquisition than concurrent reproduction in imitation learning (Weeks, Hall, \& Anderson, 1996). This result suggests that different mechanisms and processes might be involved in delayed imitation learning. In order to study these potential differences the acquisition of long movement sequences has to be studied without guiding stimuli. Our assignment algorithm allows the investigation of order errors in imitation learning for long movement sequences.

Visual spatial learning has been assessed in clinical research and diagnosis using the Corsi block-tapping task (Berch, Krikorian, \& Huha, 1998). The assessment in this task concentrates on the immediate recall memory span of visual-spatial items. In this task the stimuli, physical objects or locations on a computer screen are visible during reproduction, and the assignment is achieved either by direct physical contact with the object or by simple SNA. With SNOA the assessment can be extended to delayed imitation learning of long sequences. This assessment might reveal typical error patterns in the acquisition of metric and sequential information in patients. These error patterns may further characterize deficits and play a role in clinical diagnosis.

Imitation learning has been studied with sequences of connected pen strokes (Agam, Galperin, Gold, \& Sekuler, 2007; Sekuler, Siddiqui, Goyal, \& Rajan, 2003). Most likely this task involves different control strategies than our pen-up pointing task (Desmurget, Jordan, Prablanc, \& Jeannerod, 1997). Moreover, since previous studies focused on short pen-stroke sequences, order errors were not expected to play a significant role and were not analyzed (Agam et al., 2007). Instead, the assignment was implemented by fitting of local metric deformations of the reproduction in order to match the stimulus sequence. Such pattern-matching techniques (e.g., Burr, 1981) become problematic with inaccurately and incompletely reproduced movement sequences, especially when the number of targets is much higher than the number of reproductions (Chui \& Rangarajan, 2003). In contrast, considering the reproduction order makes the assignment robust with respect to outliers (Scott \& Nowak, 2006). Thus, even though SNOA is not expected to provide major advantages for the existing pen-stroke tasks, it may become suitable for evaluating inaccurate and incomplete reproduction of long pen-stroke sequences.

Since pattern-matching algorithms fit local deformations, they further assume that the distances between the targets and the corresponding reproductions are linked to the spatial position of the targets. Such systematic deformations may play an important role when the reproduction of the motor sequence is mediated by mechanical devices to which subjects are not fully adapted (mouse pointer, mechanical levers, etc.). However, such systematical spatial deformations probably play a minor role when pointing is performed with the hand in a highly trained working space, as in our paradigm. Even though the SNOA presented here does not consider such deformations, a combination of orderconsidering assignment and fitting of local deformations may be a useful strategy to extend the current approach.

\section{Conclusion}

With the new assignment method presented in this article the investigation of movement sequences could be extended to experimental conditions with inaccurately and incompletely reproduced movement sequences. The comparison to a simple nearest neighbor assignment showed that such an approach is necessary to evaluate these movement sequences under these conditions. The investigation of learning in such situations might induce new theoretical insights into differences between the acquisition of movement structure and the acquisition of metric information. Further, it may reveal learning deficits of patients with movement disorders or memory impairments.

Acknowledgements The study was supported by a grant from the Deutsche Forschungsgemeinschaft (GRK 1091). We thank Stefan Glasauer for encouragement and advice. We thank Ms. K. Ogston for her help in copyediting the manuscript. We thank all subjects who participated in the study for their time and effort.

\section{References}

Addis, K. M., \& Kahana, M. J. (2004). Decomposing serial learning: what is missing from the learning curve? Psychonomic Bulletin \& Review, 11, 118-124.

Agam, Y., Galperin, H., Gold, B. J., \& Sekuler, R. (2007). Learning to imitate novel motion sequences. Journal of Vision, 7, 1-17. doi:10.1167/7.5.1

Basdogan, C., Kiraz, A., Bukusoglu, I., Varol, A., \& Doğanay, S. (2007). Haptic guidance for improved task performance in steering microparticles with optical tweezers. Optics Express, 15, 11616-11621. doi:10.1364/OE.15.011616

Berch, D., Krikorian, R., \& Huha, E. M. (1998). The Corsi blocktapping task: methodological and theoretical considerations. Brain and Cognition, 38, 317-338. doi:10.1006/brcg.1998.1039

Burr, D. J. (1981). Elastic matching of line drawings. IEEE Transactions on Pattern Analysis and machine Intelligence, 3, 708-713.

Chui, H., \& Rangarajan, A. (2003). A new point matching algorithm for non-rigid registration. Computer Vision and Image Understanding, 89, 114-141. doi:10.1016/S1077-3142(03)00009-2

Desmurget, M., Jordan, M., Prablanc, C., \& Jeannerod, M. (1997). Constrained and unconstrained movements involve different control strategies. Journal of Neurophysiology, 77, 1644-1650.

Ditterich, J., Eggert, T., \& Straube, A. (1998). Fixation errors and timing in sequences of memory-guided saccades. Behavioural Brain Research, 95, 205-217. doi:10.1016/S0166-4328(97)00160-5

Ghilardi, M. F., Moisello, C., Silvestri, G., Ghez, C., \& Krakauer, J. W. (2009). Learning of a sequential motor skill comprises explicit and 
implicit components that consolidate differently. Journal of Neurophysiology, 101, 2218-2229. doi:10.1152/jn.01138.2007

Hays, A. V., Richmond, B. J., \& Optican, L. M. (1982). A UNIX-based multiple process system for real-time data acquisition and control. United States: Electron Conventions, El Segundo, CA.

Hikosaka, O., Rand, M. K., Miyachi, S., \& Miyashita, K. (1995). Learning of sequential movements in the monkey: process of learning and retention of memory. Journal of Neurophysiology, $74,1652-1661$.

Klein, K. A., Addis, K. M., \& Kahana, M. J. (2005). A comparative analysis of serial and free recall. Memory \& Cognition, 33, 833-839.

Lashley, K. S. (1951). The problem of serial order in behavior. In L. A. Jeffress (Ed.), Cerebral mechanisms in behaviour: The Hixon symposium (pp. 112-131). New York: Wiley.

Levenshtein, V. I. (1966). Binary codes capable of correcting deletions, insertions, and reversals. Soviet Physics Doklady, 10, 707-710.

Pratt, V. R. (1972). Shellsort and sorting networks. Ph.D. Thesis, Stanford University.

Schwager, M., Anderson, D. M., Butler, Z., \& Rus, D. (2007). Robust classification of animal tracking data. Computers and Electronics in Agriculture, 56, 46-59. doi:10.1016/j.compag.2007.01.002
Scott, C., \& Nowak, R. (2006). Robust contour matching via the order-preserving assignment problem. Image Processing, IEEE Transactions on, 15, 1831-1838. doi:10.1109/TIP.2006.877038

Sekuler, R., Siddiqui, A., Goyal, N., \& Rajan, R. (2003). Reproduction of seen actions: stimulus-selective learning. Perception, 32, 839-854. doi:10.1068/p5064

Shea, C. H., \& Wulf, G. (2005). Schema theory: A critical appraisal and reevaluation. Journal of Motor Behavior, 37, 85-102. doi:10.3200/JMBR.37.2.85-102.

Sternberg, S., Monsell, S., Knoll, R. L., \& Wright, C. E. (1978). The latency and duration of rapid movement sequences: Comparisons of speech and typewriting. In G. E. Stelmach (Ed.), Information processing in motor control and learning (pp. 117-152). New York: Academic.

Weeks, D. L., Hall, A. K., \& Anderson, L. P. (1996). A comparison of imitation strategies in observational learning of action patterns. Journal of Motor Behavior, 28, 348-358.

Wilde, H., \& Shea, C. H. (2006). Proportional and nonproportional transfer of movement sequences. The Quarterly Journal of Experimental Psychology, 59, 1626-1647. doi:10.1080/ 17470210500303755 